\title{
Prevalence of Plasmodium falciparum delayed clearance associated polymorphisms in adaptor protein complex 2 mu subunit (pfap2mu) and ubiquitin specific protease 1 (pfubp1) genes in Ghanaian isolates
}

\author{
Tryphena Adams', Nana Aba A. Ennuson ${ }^{1}$, Neils B. Quashie ${ }^{2,3}$, Godfred Futagbi ${ }^{1}$, Sena Matrevi²,
} Oheneba C. K. Hagan ${ }^{4}$, Benjamin Abuaku², Kwadwo A. Koram² and Nancy O. Duah ${ }^{2^{*}}$

\begin{abstract}
Background: Plasmodium falciparum delayed clearance with the use of artemisinin-based combination therapy (ACTs) has been reported in some African countries. Single nucleotide polymorphisms (SNPs) in two genes, P. falciparum adaptor protein complex 2 mu subunit (pfap2mu) and ubiquitin specific protease 1 (pfubp 1), have been linked to delayed clearance with ACT use in Kenya and recurrent imported malaria in Britain. With over 12 years of ACT use in Ghana, this study investigated the prevalence of SNPs in the pfap2mu and pfubp 1 in Ghanaian clinical $P$. falciparum isolates to provide baseline data for antimalarial drug resistance surveillance in the country.

Methods: Filter paper blood blots collected in 2015-2016 from children aged below 9 years presenting with uncomplicated malaria at hospitals in three sentinel sites Begoro, Cape Coast and Navrongo were used. Parasite DNA was extracted from 120 samples followed by nested polymerase chain reaction (nPCR). Sanger sequencing was performed to detect and identify SNPs in pfap2mu and pfubp 1 genes.

Results: In all, 11.1\% (9/81) of the isolates carried the wildtype genotypes for both genes. A total of 164 pfap2mu mutations were detected in 67 isolates whilst 271 pfubp 1 mutations were observed in 72 isolates. The majority of the mutations were non-synonymous (NS): 78\% (128/164) for pfap2mu and 92.3\% (250/271) for pfubp 1. Five unique samples had a total of 215 pfap2mu SNPs, ranging between 15 and 63 SNPs per sample. Genotypes reportedly associated with ART resistance detected in this study included pfap2mu S160N $(7.4 \%, 6 / 81)$ and pfubp 1 E1528D $(7.4 \%, 6 / 81)$ as well as D1525E $(4.9 \%, 4 / 81)$. There was no significant difference in the prevalence of the SNPs between the three ecologically distinct study sites (pfap2mu: $X^{2}=6.905, d f=2, P=0.546 ;$; fubp $1: X^{2}=4.883, d f=2, P=0.769$ ).

Conclusions: The detection of pfap2mu and pfubp 1 genotypes associated with ACT delayed parasite clearance is evidence of gradual nascent emergence of resistance in Ghana. The results will serve as baseline data for surveillance and the selection of the genotypes with drug pressure over time. The pfap2mu S160N, pfubp1 E1528D and D1525E must be monitored in Ghanaian isolates in ACT susceptibility studies, especially when cure rates of ACTs, particularly $\mathrm{AL}$, is less than $100 \%$.
\end{abstract}

Keywords: Plasmodium falciparum, Antimalarial drug resistance, Artemisinin, ACT, pfubp1, pfap2mu, Mutations, Ghana

\footnotetext{
* Correspondence: nduah@noguchi.ug.edu.gh

${ }^{2}$ Department of Epidemiology, Noguchi Memorial Institute for Medical

Research, College of Health Sciences, University of Ghana, Accra, Ghana

Full list of author information is available at the end of the article
} 


\section{Background}

Malaria is still a debilitating disease, especially in subSaharan Africa (sSA) where there were 212 million cases and 429,000 malaria-related deaths in 2015 [1]. There has been a 21 and $29 \%$ reduction in morbidity and mortality, respectively, since 2010, probably as a result of the implementation of integrated control strategies [1, 2]. The control efforts employed included the use of insecticidetreated mosquito nets (ITNs), indoor residual spraying (IRS), chemoprevention in pregnant women and children as well as chemotherapy with artemisinin-based combination therapy (ACT). As such, the development of Plasmodium falciparum resistance to artemisinin (ART) derivatives as reported from Southeast Asia (SEA) is quite worrying [1]. Chemotherapy, which is one of the core control strategies for the disease, has been hindered over the years by the emergence and spread of parasites resistant to the commonly used antimalarial drugs [1]. Currently, the World Health Organisation (WHO) has initiated the containment of drug resistance in the SEA region with the deployment of a multi-sector strategy [1]. Although this initiative is commendable, the need for country-level monitoring of the genome of parasite populations for possible evolution and selection due to drug pressure is also crucial for the early detection of emerging drug resistance.

For over a decade, molecular markers of antimalarial drug resistance have been used to monitor the emergence and spread of drug resistance in malaria endemic areas. These genetic markers are mainly single nucleotide polymorphisms in genes encoding drug target proteins in essential biochemical pathways of the parasite. The levels of drug susceptibility in the parasites have been linked to SNPs or haplotypes of the genes, and these markers are relevant in antimalarial drug efficacy studies. The recent observation of parasite resistance to ART in SEA set into motion the need to discover a molecular marker for surveillance of drug susceptibility. Ariey et al. [3] discovered the SNPs in the kelch propeller domain on chromosome 13 of the P. falciparum genome known as $\mathrm{k} 13$ in drug resistance isolates in vitro. Three of the k13 polymorphisms, C580Y, R538T and $\mathrm{Y} 493 \mathrm{H}$ were also present in slow clearing clinical isolates with ART use. The presence of the SNPs showed varying parasite clearance half-life in patients; however, the C580Y mutation was linked to longest parasite clearance half-life compared to the other SNPs. So far these SNPs have not been detected in African isolates [4-6] and the quest for novel markers for ART resistance is ongoing. Henriques et al. [7] linked SNPs in two genes, the P. falciparum adaptor protein complex $2 \mathrm{mu}$ subunit (pfap2mu) and ubiquitin-specific protease 1 ( $p f u b p 1)$ to delayed clearance of parasites. The pfap $2 m u$ gene mutation was at codon 160 resulting in the amino acid change from serine to either asparagine or threonine (S160N/T). The pfubp1 gene mutations were at codon 1525, a change from aspartic acid to glutamic acid (D1525E) and codon 1528 from glutamic acid to aspartic acid (E1528D) in the Kenyan isolates. In addition, Sutherland et al. [8] reported four UK residents with imported malaria who showed recurrent malaria after AL treatment [8]. These mutations, pfap2mu S160N and pfubp1 E1525D/Q were observed in the recurrent parasites but none of the known $\mathrm{K}-13$ gene mutations were observed [8]. Although the role played by both genes in artemisinin action is not clearly understood, the ap $2 m u$ gene is known to encode the $\mu$ - subunit of the adaptor protein 2 complex (AP2) involved in clathrin-mediated endocytosis into the parasite vacuole [9]; $u b p 1$ encodes a deubiquitinating (DUB) enzyme that functions by cleaving ubiquitin from any protein or peptide to which it is joined [10]. The polymorphic homologues of these two P. falciparum genes were first identified in the rodent malaria parasite, $P$. chabaudi ( $p c u b p 1$ encodes ubiquitin carboxyterminal hydrolase 1) and pcap $2 m u$ encodes clathrin vesicle-associated adaptor $2 \mathrm{mu}$ subunit), as being associated with ART resistance [11]. More studies are therefore needed to validate these polymorphisms and their role in antimalarial drug resistance.

The use of ACTs in Ghana began in 2005 and since then the cure rate of the drugs in use, artesunate-amodiaquine (AS-AQ) and artemether-lumifantrine (AL), has been 100 and $97.6 \%$, respectively, as of 2014 [12]. Surveillance studies using the $P$. falciparum multidrug resistance gene (pfmdr1) SNPs (haplotype N86-F184-D1246) linked to reduced parasite susceptibility to AL showed an increasing trend over the years in Ghana from 2005 to 2010 [13]. In addition, increased pfmdr1 gene copy number linked to parasite reduced susceptibility to artesunate (AS), mefloquine (MQ), halofantrine and AL [14-16] were also detected in Ghanaian isolates [13]. The findings from the reported studies above are indicative of a subtle emergence of parasite resistance to ART and to ACTs especially AL in Ghana. Therefore, the monitoring of newly discovered molecular markers is essential as an early warning signal to the emergence of resistance in Ghana. This study determined the prevalence of known and novel SNPs in the $p f u b p 1$ and $p f a p 2 m u$ in Ghanaian isolates collected from three ecologically distinct areas for monitoring antimalarial drug efficacy in Ghana to serve as baseline data for antimalarial drug resistance surveillance in Ghana.

\section{Methods}

\section{Study sites}

The Noguchi Memorial Institute for Medical Research (NMIMR) in collaboration with the National Malaria Control Programme (NMCP) have set up ten sentinel sites in the ten regions of Ghana for monitoring antimalarial drug efficacy. These sites lie in the three distinct ecological zones in the country. Of the ten sites, samples 
from three sites were used for this study. The sites include Navrongo $\left(10^{\circ} 53^{\prime} 44.05^{\prime \prime} \mathrm{N}, 1^{\circ} 05^{\prime} 31.56^{\prime \prime W}\right)$ located in the Kassena Nankana District in the Upper East Region and lies in the guinea savannah zone; Begoro $\left(6^{\circ}\right.$ $\left.23^{\prime} 29.76 " \mathrm{~N}, 0^{\circ} 22^{\prime} 46.20^{\prime \prime W}\right)$ located in the Fanteakwa District of the Eastern Region lies in the forest zone; Cape Coast $\left(5^{\circ} 06^{\prime} 00^{\prime \prime} \mathrm{N}, 1^{\circ} 15^{\prime} 00^{\prime \prime} \mathrm{W}\right)$, the capital town of Central Region lies in the coastal savannah zone (Fig. 1). The forest and coastal savannah zones experience perennial malarial transmission whilst the guinea savannah zone experiences a seasonal malaria transmission pattern with almost all cases occurring during rainy months between May-June and October-November of each year.

\section{Study samples}

Filter paper blood blots collected in 2015-2016 from children aged below 9 years with uncomplicated malaria reporting at designated health care facilities in Navrongo, Begoro and Cape Coast were used for the study. In total 120 samples (40 from each site) were used for this investigation.

\section{Detection of pfubp1 and pfap2mu gene polymorphisms}

Parasite DNA was extracted from 120 pre-treatment blood blot samples on filter paper (Whatman ${ }^{\mathrm{TM}} 3$ Little Chalfont, United Kingdom) using the QIAmp DNA mini kit (Qiagen $\mathrm{GmBH}$, Hilden, Germany) as per the manufacturer's protocol. This was followed by amplification of the $p f a p 2 m u$ and $p f u b p 1$ genes using nested polymerase chain reaction (nPCR) following a previously published protocol [7] with minor modifications. The PCR was performed in a total volume of $25 \mu \mathrm{l}$ with the following reaction mixture: $0.2 \mu \mathrm{M}$ of each primer (Table 1), 4.0 $\mathrm{mM} \mathrm{MgCl}_{2}, \quad 0.4 \mu \mathrm{M}$ deoxynucleotides triphosphate (dNTPs), 1 U One-Taq polymerase (New England Biolabs, Massachusetts, USA), $1 \times$ PCR buffer, nuclease free water and $2 \mu \mathrm{l}$ of the extracted parasite DNA. One microlitre of the first round product was used as a template in a $50 \mu \mathrm{l}$ inner PCR reaction. A DNA sample extracted from the 3D7 parasite strain was used as a positive control. The PCR thermal conditions were the same for both genes but different annealing temperatures as shown in Table 1 . The

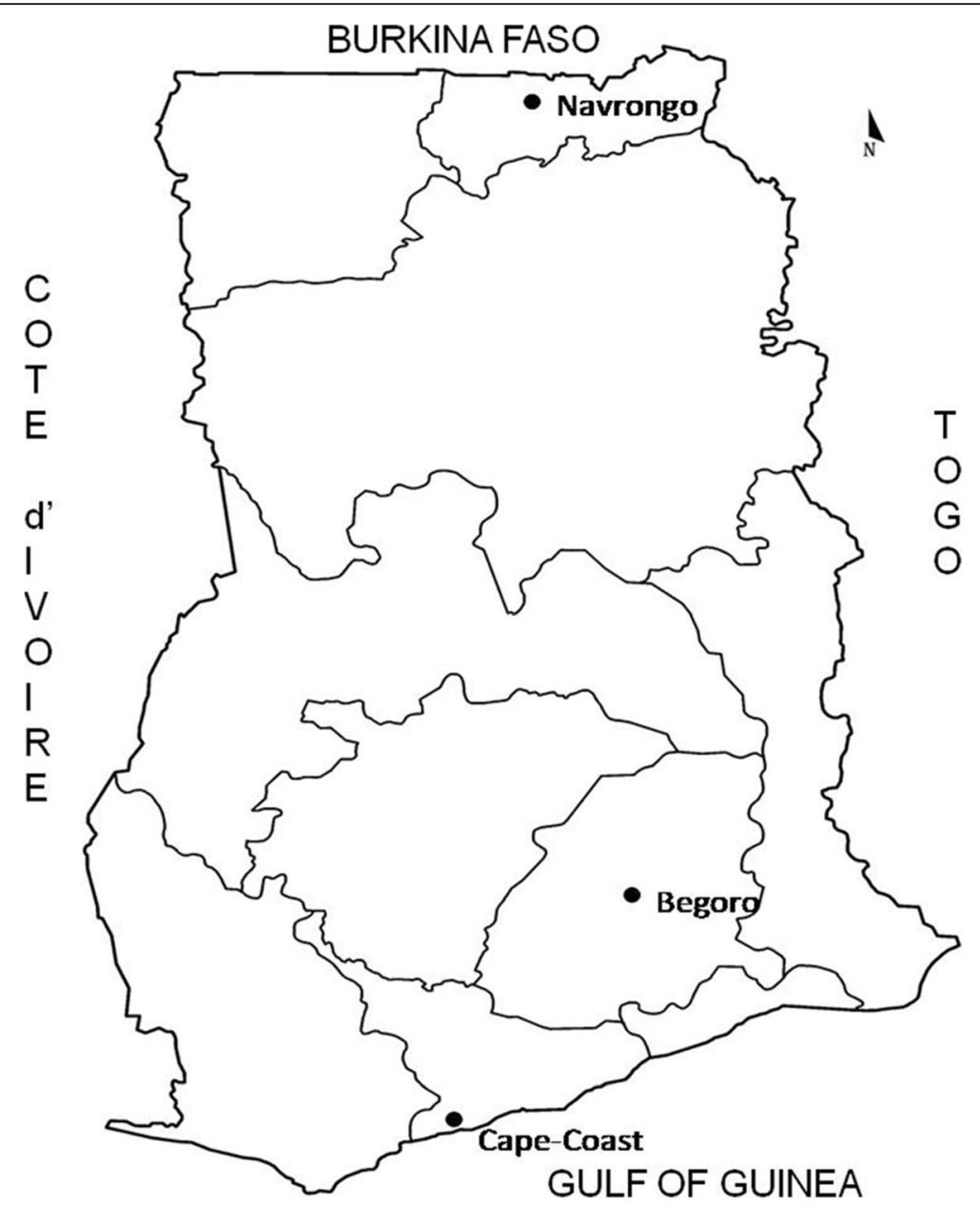

Fig. 1 The map of Ghana showing the three study sites, Navrongo, Begoro and Cape Coast in three different ecological areas, guinea savannah, forest and coastal savanna 
Table 1 Primer sequences, sizes of PCR amplicons and annealing temperature of the amplification of pfap2mu and pfubp $1 \mathrm{genes}$

\begin{tabular}{|c|c|c|c|}
\hline Gene & Primers $\left(5^{\prime}-3^{\prime}\right)$ & Size of PCR amplicon (bp) & Annealing temperature $\left({ }^{\circ} \mathrm{C}\right)$ \\
\hline \multirow[t]{6}{*}{ pfap2mu } & Primary amplification & 2247 & 50 \\
\hline & Forward: AAGACTGTCAAATGTAAAAGACCC & & \\
\hline & Reverse: CTCATGTAAAACAAAAAGTGAGG & & \\
\hline & Secondary amplification & 841 & 52 \\
\hline & Forward: GATATCCACAAACATTAGAAGTG & & \\
\hline & Reverse: CCATCTGGTGGTGTGAAGG & & \\
\hline \multirow[t]{6}{*}{ pfubp 1} & Primary amplification & 484 & 52 \\
\hline & Forward: CGCCCGTACTATGAAGAAGATC & & \\
\hline & Reverse: GGCTITTACCTGAACTGTTCAGG & & \\
\hline & Secondary amplification & 304 & 57 \\
\hline & Forward: CGTAAACAGAATATTCAGGATTGC & & \\
\hline & Reverse: CTAGCCCTITATTATCATTATCG & & \\
\hline
\end{tabular}

thermal cycle programme for each 1st amplification was $94{ }^{\circ} \mathrm{C}$ for $3 \mathrm{~min}$, and 30 cycles of $94{ }^{\circ} \mathrm{C}$ for $30 \mathrm{~s}$, annealing temperature for $30 \mathrm{~s}$ and $68^{\circ} \mathrm{C}$ for $1 \mathrm{~min}$ with a final extension of $68{ }^{\circ} \mathrm{C}$ for $15 \mathrm{~min}$. The second round of PCR consisted of initial denaturation at $94{ }^{\circ} \mathrm{C}$ for $3 \mathrm{~min}$, followed by 40 cycles of $94{ }^{\circ} \mathrm{C}$ for $30 \mathrm{~s}$, annealing temperature for $30 \mathrm{~s}$ and $68{ }^{\circ} \mathrm{C}$ for $45 \mathrm{~s}$ with a final extension of $68{ }^{\circ} \mathrm{C}$ for $10 \mathrm{~min}$. The PCR amplicons for the fragments of the two genes were sequenced using Sanger sequencing.

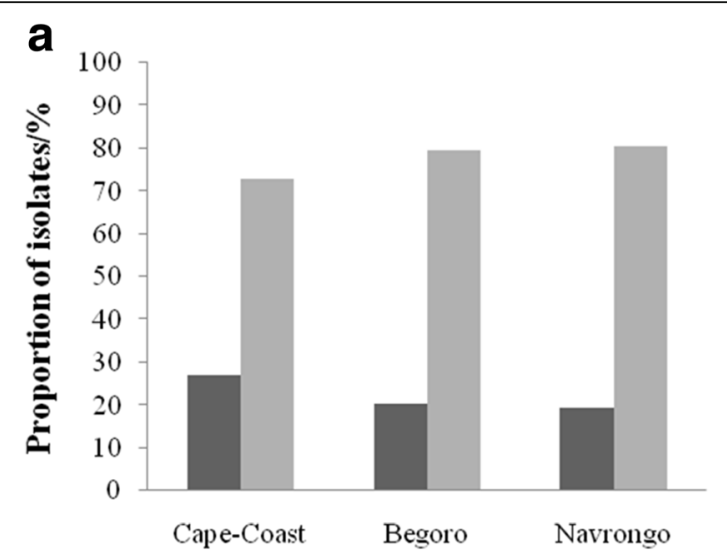

- Synonymous

घ Nonsynonymous

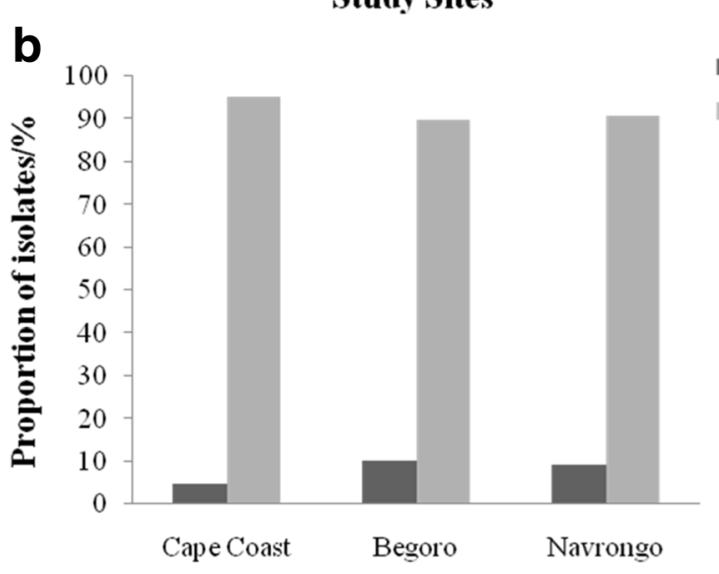

Study sites

Fig. 2 Distribution of pfap2mu and pfubp1 NS and SYN mutations in isolates from the three sites. a pfap2mu. b pfubp 1 


\section{Data analysis}

The sequence data of the isolates were analysed using the CLC Genomics Workbench 10.01 software (Qiagen, Aarhus, Denmark) and Benchling.com (California, CA, USA). PF3D7_1218300 and PF3D7_0104300 (PlasmoDB) were used as reference sequences to detect SNPs in the pfap $2 m u$ and $p f u b p 1$ respectively. Poor quality sequences of isolates after three sequencing trials were not analysed. The prevalence of individual SNPs was determined for each site. Chi-square tests were used to compare the proportions of mutations occurring in the three sites and to determine any significant differences in the prevalence of the mutations among the three sites using the GraphPad Prism 5 (GraphPad Software Inc, La Jolla, CA, USA). Statistical significance was defined as a $P$-value $\leq 0.05$.

\section{Results}

\section{Polymorphisms in pfap2mu and pfubp 1}

For the pfap $2 m u$ gene, 96 samples were sequenced and 15 were of low quality as determined by quality assurance analysis. Of the 81 good sequences, 35\% (28/ $81), 35 \%(28 / 81)$ and $31 \%(25 / 81)$ were from CapeCoast, Begoro and Navrongo, respectively. The proportion of isolates with no mutations in the pfap $2 m u$ gene, that is wildtype sequence as the 3D7 strain, was $11.1 \%$ $(9 / 81)$. The sequence analysis revealed several SNPs and the total number observed in 67 samples (of the 72 with mutations) was 164 SNPs with $\leq 5$ mutations per sample. Of the 164 SNPs, 78.0\% (128/164) were nonsynonymous (NS) and $22.0 \%(36 / 164)$ were synonymous (SYN) mutations. The distribution of pfap2mu NS and SYN mutations in isolates from the three sites is shown in Fig. 2a. There was no significant difference in the type of mutation (SYN or NS) present between the three sites $\left(\chi^{2}=1.960, d f=2, P=0.360\right)$. The distribution of the single or multiple mutations for each site is shown in Fig. 3a. About $68.6 \%(46 / 67)$ of the isolates with mutations had more than one mutation and $36 \%(9 / 25)$ of the isolates from Navrongo had more than three mutations per isolate. In addition, there were insertions and
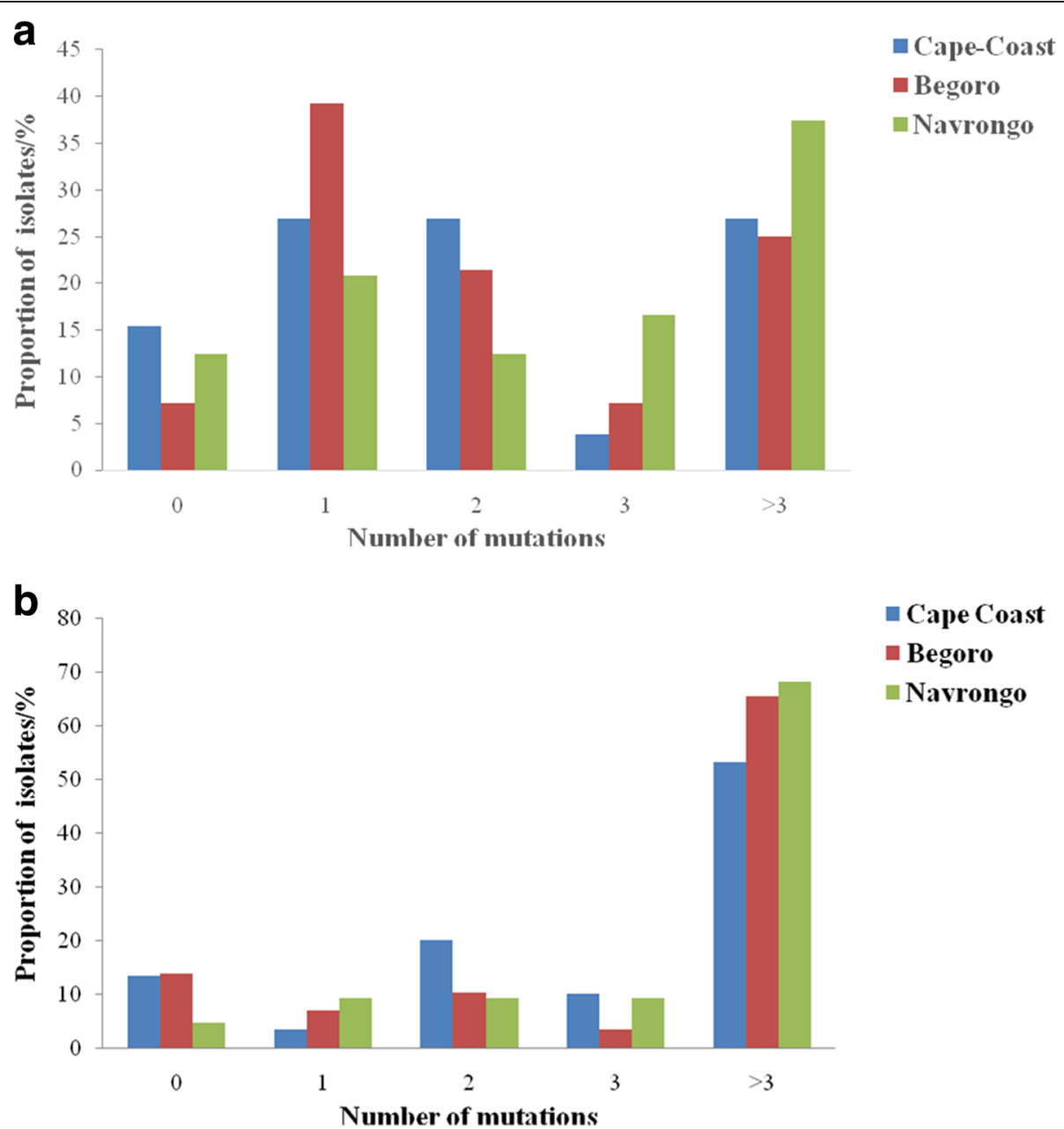

Fig. 3 Proportion of isolates from the three sites with varying number of pfap2mu and pfubp 1 mutations. a pfap $2 m u$. b pfubp 1 
deletions in some of the isolates from the three sites. In all 22 common SNPs were detected in either two of the three sites or all three sites. These include Q149R, S160N, V161K, V161E, D168E, R188R, D203F, D203Y, E206*, T235T, N240Y, N240F, K256*, D263V, V270V, I272I, G284G, K285E, T302T, N317S, T318T and T325T. Nine of these SNPs were shared in all three sites and the proportion of isolates from the study sites is shown in Fig. 4a. Of these SNPs, three NS mutations were present in isolates from the three sites: S160N, D168E and V161K. The nucleotide changes for the SNPs are shown in Table 2. The amino acid sequences alignment for 21 isolates are shown in Fig. 5. There were 5 other isolates (of the 72 with mutations) with a total of 215 pfap $2 m u$ SNPs (G020, 63 SNPs; G022, 60 SNPs; G025, 47 SNPs; G029, 30 SNPs; G034, 15 SNPs) and were all from Begoro. The amino acid sequences for these 5 isolates are shown in Fig. 6.
For the $p f u b p 1$ gene, 81 quality sequence data were analysed comprising 37\% (30/81), 35.8\% (29/81) and $27.2 \%$ (22/81) from Cape Coast, Begoro and Navrongo, respectively. About $11.1 \%$ (9/81) of the isolates had no mutations in their pfubp1 gene. A total of 271 SNPs were observed in the 72 sequences of which $92.3 \%$ (250/ 271) were NS and $7.7 \%(21 / 271)$ were SYN mutations. The proportion of isolates with either pfubp1 NS or SYN mutations for each site is shown in Fig. 2b. The proportion of isolates with varying number of mutations is also shown in Fig. 3b for the three study sites. Overall, 93.1\% (67/72) of the isolates with mutations had more than one pfubp1 SNP. The majority of isolates from Begoro $(65.5 \%, 19 / 29)$ had more than 3 mutations per isolate for the $p$ fubp 1 gene. There were 15 common SNPs detected in isolates from all three sites. These include D1539D, E1528D, I1487I, I1552M, K1502*, K1537L, N1542D, N1551G, N1560I, N1560K, P1547P, Q1543H, Y1501F,

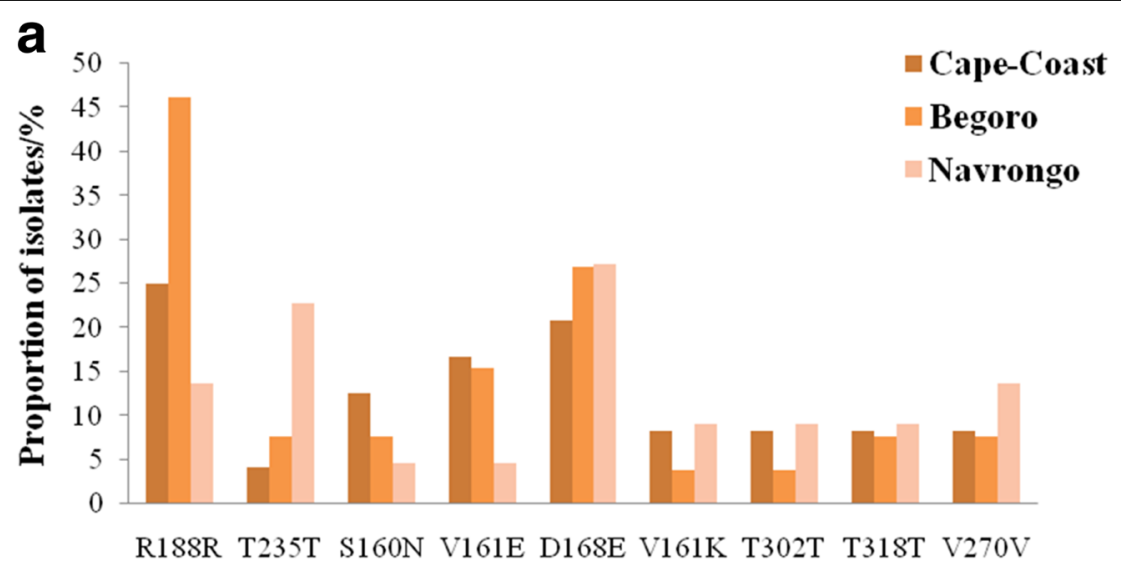

Mutation

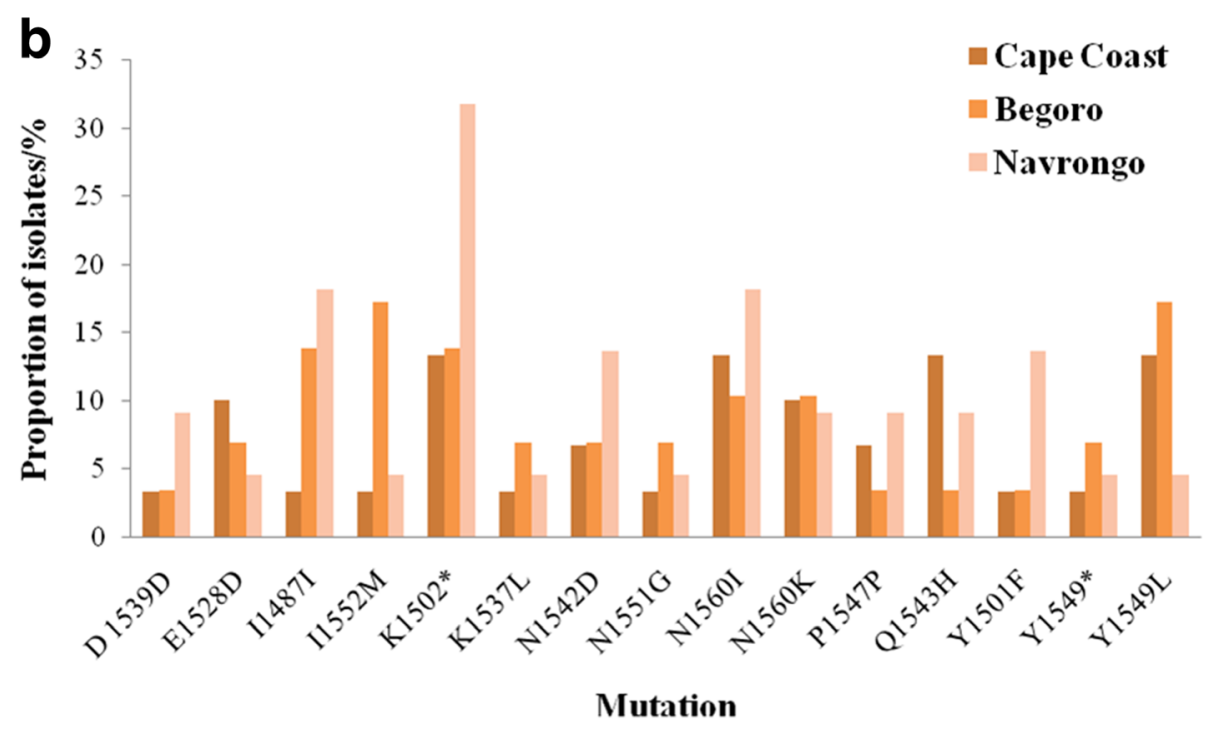

Fig. 4 Proportion of isolates from the three sites with shared pfap $2 m u$ and $p f u b p 1$ mutations. a pfap $2 m u$. b pfubp 1 
Table 2 Shared pfap2mu mutations observed in the isolates from the three sites. Mutations indicated in bold are known delayed clearance genotypes, underlined nucleotides are the changed bases

\begin{tabular}{|c|c|c|}
\hline Nucleotide position & Nucleotide change & $\begin{array}{l}\text { Amino acid position } \\
\text { and change }\end{array}$ \\
\hline 446 & CAG to $\underline{C G G}$ & Q149R \\
\hline 479 & AGGT to AAT & $\mathrm{S} 160 \mathrm{~N}$ \\
\hline 481 & $\underline{G} T G$ to $\underline{A A G}$ & V161K \\
\hline 482 & GIG to GAG & V161E \\
\hline 504 & GAT to GAA & D168E \\
\hline 564 & $A G \underline{A}$ to $A G \underline{G}$ & $\mathrm{R} 188 \mathrm{R}$ \\
\hline 607 & $\underline{\text { GAT to }} \underline{\mathrm{T}} \mathrm{AT}$ & D203Y \\
\hline 607,608 & GAT to III & D203F \\
\hline 616 & $\underline{G} A A$ to $\underline{T} A A$ & $\mathrm{E} 206^{\mathrm{a}}$ \\
\hline 705 & $A C \underline{A}$ to $A C \underline{G}$ & $\mathrm{~T} 235 \mathrm{~T}$ \\
\hline 718 & $\underline{A}$ AT to $\underline{T}$ AT & N240Y \\
\hline 767 & $\underline{A} A G$ to $\underline{T} A G$ & $\mathrm{~K} 256^{\mathrm{a}}$ \\
\hline 789 & GAT to GIT & D263V \\
\hline 810 & GTA to GTI & V270V \\
\hline 852 & GGA to GGG & G284G \\
\hline 855 & $\underline{A A G}$ to $\underline{G} A G$ & K285E \\
\hline 951 & $A \underline{A C}$ to $A \underline{G} C$ & N317S \\
\hline 954 & $A C A$ to $A C \underline{C}$ & T318T \\
\hline
\end{tabular}

${ }^{\mathrm{a}}$ Stop codon
Y1549* and Y1549L. The proportion of isolates with these mutations from each of the study sites is shown in Fig. 4b. There were 36 other shared SNPs detected in isolates from two out of the three sites. The nucleotide changes for the commonly shared mutations are also shown in Table 3. The amino acid sequence alignment is shown in Fig. 7.

Two samples, G005 and C329, had wildtype sequences for both $p f a p 2 m u$ and $p f u b p 1$. Of the 5 isolates with many pfap2mu SNPs, 2 were wildtype for $p f u b p 1$ (G022 and G034). For the other three, G020 had 4 SNPs, G025 had 2 SNPs and G029 had 11 SNPs for the pfubp1.

\section{Prevalence of pfap2mu and pfubp1 SNPs from the three sentinel sites}

The pfap $2 m u$ SNPs were detected in 92.9\% (26/28), $85.7 \%(24 / 28)$ and 88\% (22/25) from Begoro, Cape Coast and Navrongo respectively. There was no significant difference in the prevalence of pfap $2 m u$ SNPs in the isolates from the three sites $\left(\chi^{2}=6.905, d f=2, P=0.546\right)$. The most prevalent mutation was R188R and was observed in $25.9 \%(21 / 81)$ of the isolates. The D168E was also observed in $22.2 \%(18 / 81)$ of the isolates. S160N which have been reported to be associated with ACT delayed clearance was prevalent in $7.4 \%(6 / 81)$ of the isolates whilst the V161K was seen in $6.2 \%(5 / 81)$. The S160N genotypes occurred mostly in isolates from Cape Coast $(24.7 \%)$ as compared to the other two sites. In all, 35 indels were identified with $17.1 \%$ (6/ 35 ) causing a frame shift in the sequence reading frame (Fig. 5).

For pfubp1, mutations were detected in $86.7 \%(26 / 30)$ of the Cape Coast isolates, $86.2 \%$ (25/29) for Begoro and

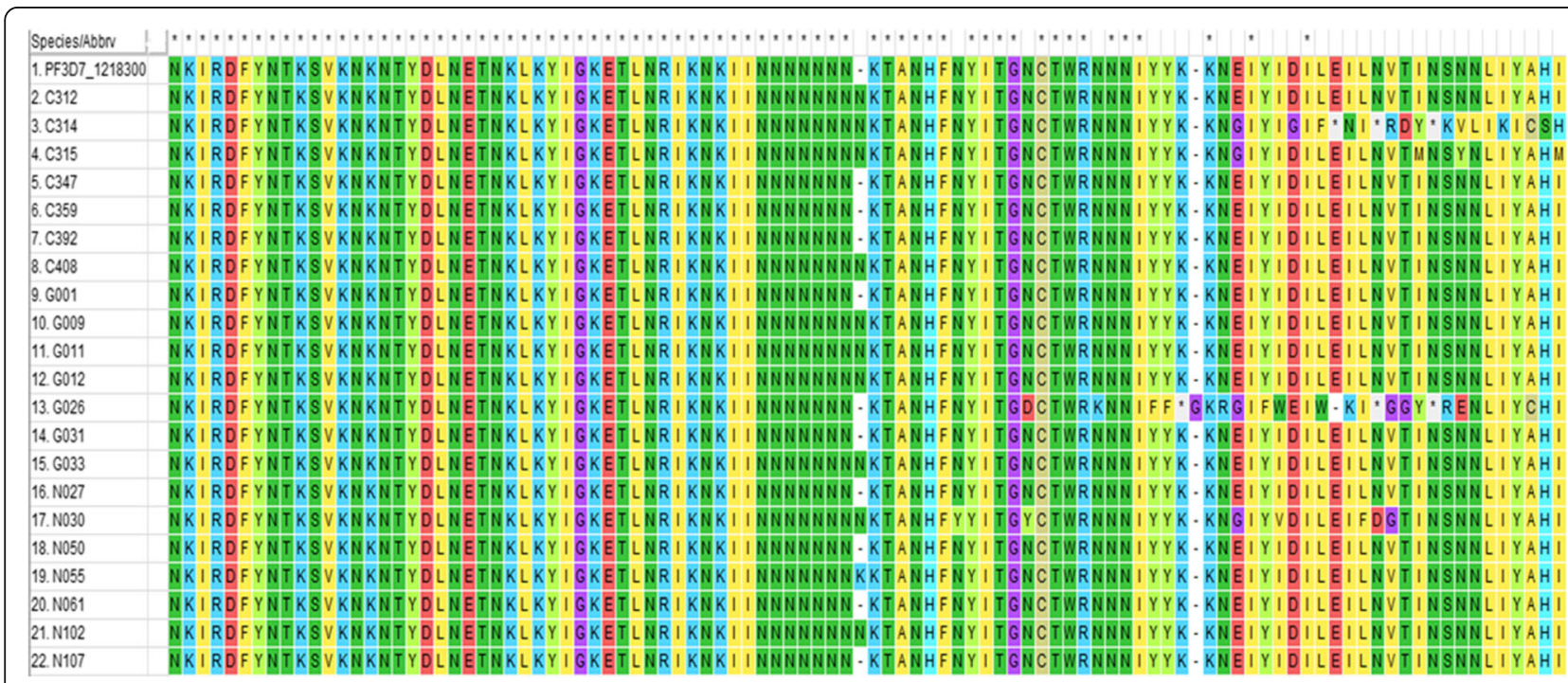

Fig. 5 A sequence alignment of pfap2mu gene showing amino acid changes due to single nucleotide polymorphisms. The alignment was done using pfap2mu reference sequence of the 3D7 strain (PF3D7_1218300). Mutations present at codons 185-282, nucleotide positions 553-849 of pfap2mu for 21 samples. Samples C314 and G026 had a frameshift, samples C312. C314, C315, C408, G009, G011, G012, G033, N030 and N102 had an asparagine $(\mathrm{N})$ insertion at codon 233, as well as a lysine $(K)$ insertion at the same position for sample N055 


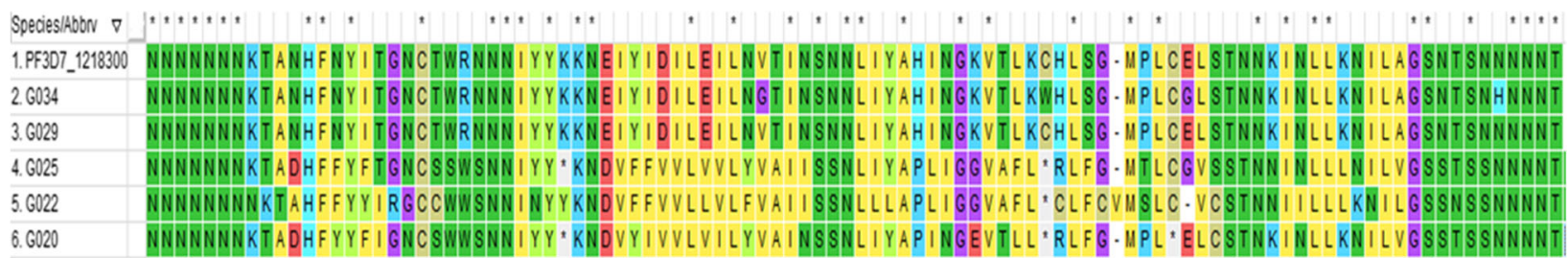

Fig. 6 A sequence alignment for 5 samples with multiple mutations. The alignment was done using pfap2mu reference sequence of the 3D7 strain (PF3D7_1218300). About 215 SNPs were observed in these isolates ranging from 15 to 63 SNPs per isolate. These mutations were found from codons 227-324, nucleotide positions 679-970 and are likely due to multiplicity of infection
95.5\% (21/22) for Navrongo. There was no significant difference in the prevalence of SNPs in the isolates from the three sites $\left(\chi^{2}=4.883, d f=2, P=0.769\right)$. The most prevalent NS mutation was K1502* which was observed in $18.5 \%(15 / 81)$ of the isolates. The reported SNPs linked to delayed clearance of parasite with ACT use, E1528D and D1525E, were observed in 7.4\% (6/81) and $4.9 \%(4 / 81)$ of the isolates, respectively. The predominant SYN mutation I1487I was observed in $11.1 \%(9 / 81)$ of the isolates. In all, 20 indels were identified with $25 \%$ $(5 / 20)$ causing a frame shift in the sequence reading frame (Table 4).

The results showed a number of SNPs that were being inherited together on the gene as haplotypes in some of the isolates. However, the prevalence of the haplotypes was low (Table 5). Twenty-two different haplotypes were observed: 3 for $p f a p 2 m u$ and 19 for $p f u b p 1$. The most prevalent haplotype for the pfap $2 m u$ gene was V161KD168E, which was observed in $8.6 \%(7 / 81)$ of the isolates with mutations. For the pfubp1 gene, the haplotypes Y1548L-N1560I, Y1549L-I1552M and N1560I-L1563 were each observed in $3.7 \%(3 / 81)$ of the isolates. Most of the haplotypes for both genes were observed in isolates from Begoro.

\section{Discussion}

The search for a molecular marker for the early detection of ART resistance by the malaria parasite is ongoing. The use of molecular markers to track and identify early development of parasite resistance to drugs is a powerful tool that should be available in all malarious regions of the world. With the implementation of ACTs in Africa, studies to identify possible markers of resistance have not been conducted extensively in the continent. The key $k 13$ molecular marker, which has been linked to drug resistance in SEA has not been observed in African isolates, partly because there is no 'true resistance' to ARTs except delayed clearance of parasites. New markers that have recently been discovered, such as the pfap2mu and pfubp1 gene mutations [7], need further validation for their role in delayed parasite clearance. This study detected ART delayed clearance associated polymorphisms of the pfap $2 m u$ and $p f u b p 1$ genes in Ghanaian isolates from three sites located in three distinct ecological zones. Majority of the isolates had mutations of both genes and were mostly NS mutations. The known delayed clearance genotypes, pfap $2 m u$ N160 and pfubp1 D1528 and E1525, were observed in $7.4,7.4$ and $4.9 \%$ of the isolates, respectively. It is interesting to note that although these were not the predominant mutations, it is an indication of the presence of these drug resistance genotypes in Ghanaian parasite populations and in the long term their selection with drug use will enhance the emergence of ART resistance in Ghana.

The observation that minority of the isolates were wildtype with no mutations like the reference 3D7 strain (11.1\% for both pfap $2 m u$ and $p f u b p 1)$ is indicative of the high rate of spontaneous mutations in the two genes. For $p f a p 2 m u$, a study by Henriques et al. [7] reported that $41.5 \%$ of Kenyan isolates had wildtype gene sequence whilst another study reported $92.8 \%$ and $30.9 \%$ for Ethiopian and Tanzanian isolates, respectively [17]. Comparatively, the Ghanaian isolates had low levels of wildtype strains portraying a rapid genetic recombination of different parasite clones (multiclonal infections observed in Ghana) during the sexual stage in the vector resulting in gene shuffling [18]. About $64 \%$ and $93 \%$ of the isolates, respectively, had more than one mutation for the $p f a p 2 m u$ and $p f u b p 1$ genes.

The results revealed a high proportion of NS mutations, $78 \%$ and $92 \%$ for $p f a p 2 m u$ and $p f u b p 1$, respectively, in the Ghanaian isolates. The NS mutations are of much importance because each amino acid substitution may affect protein conformation and function [19]. Most of the NS mutations were single base or double base substitutions. For SYN mutations, it was initially assumed that since the resultant change of the nucleotide does not affect the amino acid, the change may go undetected as the gene function may not necessarily be affected [20]. However, this perception has since changed due to the evidence that SYN mutations in the pfmdr1 gene resulted in alterations in the functions of the P-glycoprotein (P-gp), a product which affects drug 
Table 3 Shared pfubp 1 mutations observed in the isolates from the three sites. Mutations indicated in bold are known delayed clearance genotypes

\begin{tabular}{|c|c|c|c|c|c|}
\hline & \\
\hline Nucleotide position & Nucleotide change & $\begin{array}{l}\text { Amino acid position } \\
\text { and change }\end{array}$ & Nucleotide position & Nucleotide change & $\begin{array}{l}\text { Amino acid position } \\
\text { and change }\end{array}$ \\
\hline 4383 & $\underline{C} \underline{C}$ to $\underline{A C} \underline{C}$ & P1461T & 4656 & ATI to AT $\underline{G}$ & I1552M \\
\hline 4386 & TAT to TAㅗ & Y1462L & 4659 & AAT to AAC & N1553N \\
\hline 4389 & $\underline{\mathrm{CGT}}$ to $\underline{\mathrm{TCG}}$ & R1463S & 4667 & TAC to TGC & Y1556C \\
\hline 4392 & $\underline{A A A}$ to $\underline{T} A A$ & $\mathrm{~K} 1464^{\mathrm{a}}$ & 4674 & $\underline{A A T}$ to $\underline{G} A T$ & N1558D \\
\hline 4461 & ATA to ATC & |11487| & 4679 & AAT to ATA & N1560l \\
\hline 4461 & ATA to ACC & |1487T & 4680 & AAT to AAA & N1560K \\
\hline 4466 & ATG to ACG & M1489T & 4683 & $\mathrm{AAA}$ to $\mathrm{AAG}$ & K1561K \\
\hline 4502 & TAT to TIT & Y1501F & 4692 & GAG to $G A \underline{C}$ & E1564D \\
\hline 4504 & $\underline{A} A A$ to $\underline{T} A A$ & $\mathrm{~K} 1502^{\mathrm{a}}$ & 4695 & $\underline{\text { ITC }}$ to $\underline{\mathrm{GGC}}$ & F1565G \\
\hline 4509 & AAT to ATI & N1503I & 4694 & $\underline{\Pi T C}$ to $\underline{\text { CAA }}$ & F1565Q \\
\hline 4509 & AAT to ATG & N1503M & 4765 & $\underline{\text { AAA }}$ to $\overline{\text { TAA }}$ & $\mathrm{K} 1589^{\mathrm{a}}$ \\
\hline
\end{tabular}

4527

4554

4557

4561

4575

4581

4581

4584

4599

4602

4608

4611

4609

4611

4617

4623

4623

4626

4629

4632

4632

4632

4641

4647

4647

4646

4650

4650

4651

4653

4653
GAA to GAC

$\underline{A A C}$ to $\underline{T}-\underline{-}$

GAA to GAC

TAT to AAT

GAC to GA-

TAT to TII

TAT to AAT

GAA to GAC

TAT to TAC

GAT to GAE

TAC to TCC

AAA to $\underline{\Pi T}$

AAA to $\underline{\Pi A}$

AAA to TAC

GAT to GAC

$\underline{A A A}$ to $C T$

AAA to AAT

AAT to GAT

CAA to CAT

CAT to $\mathrm{C}$ T

CAT to CET

AAA to AAT

CCA to CCI

TAI to TAG

TAT to TII

TAT to TI

GAT to ATT

GAT to AAT

AAT to GGT

AAT to ATT

AAT to $\underline{C T}$
E1509D

N1518Y

E1519D

Y1521N

D1525E

Y1527F

Y1527N

E1528D

Y1533Y

D1534E

Y1536S

K1537F

K1537L

K1537Y

D1539D

K1541L

K1541N

N1542D

Q1543H

H1544L

H1544P

K1544N

P1547P

Y1549 ${ }^{a}$

Y1549F

Y1549L

D1550l

D1550N

N1551G

N1551I

N1551L
Table 3 Shared pfubp 1 mutations observed in the isolates from the three sites. Mutations indicated in bold are known delayed clearance genotypes (Continued)

interactions [21]. A high prevalence of novel ap2mu mutation, D168E (25\%), was observed in the Ghanaian isolates from all three sites followed by V161K (7\%). The codons for the common mutations found among the isolates from all the three sites were between codons 160 and 170. The known SNP, pfap2mu S160N, found in Kenyan isolates with delayed clearance [7] was also found in all three sites (7.2\%). In addition, isolates from a UK patient who arrived from Angola and failed AL treatment had the S160N thereby strengthening the role of that mutation in recurrent parasitemia with $\mathrm{AL}$ use [8]. The observed pfubp1 E1528D in Ghanaian samples was also seen in Kenyan and Tanzanian isolates [7, 17]. Borrmann et al. [22] first identified this mutation in Kenyan isolates. The prevalence of E1528D in Ghanaian isolates was $7.4 \%$, which is lower than that of Kenya's $17.1 \%$ but higher than that of Tanzania $(4.8 \%)$ [7, 17]. However, with the continuing use of ACTs, the mutation may be selected and the prevalence may increase with time as observed in Kenya. The SYN mutation N1518 found in the Kenyan and Burkinabe isolates was also seen in one of the Ghanaian isolates from Begoro. Pre- and post-treatment samples from Burkina Faso, had the D1525E mutation which was seen in four Ghanaian isolates. Henriques et al. [7] compared South East Asian and African phenotypes of the pfubp1 gene and detected significant differences in the genetic signatures. The recent implication of SNPs in these genes, especially pfap $2 m u \mathrm{~S} 160 \mathrm{~N}$ and pfubp 1 E1528D and D1525E in ART resistance, raises a genuine concern due to their presence in Ghanaian isolates.

Of the three sites, Begoro (forest) had the most pfap2mu mutations (93\%) followed by Cape-Coast (coastal savannah) (86\%) and Navrongo (guinea savannah) (88\%). Despite this observation, there is comparatively higher 


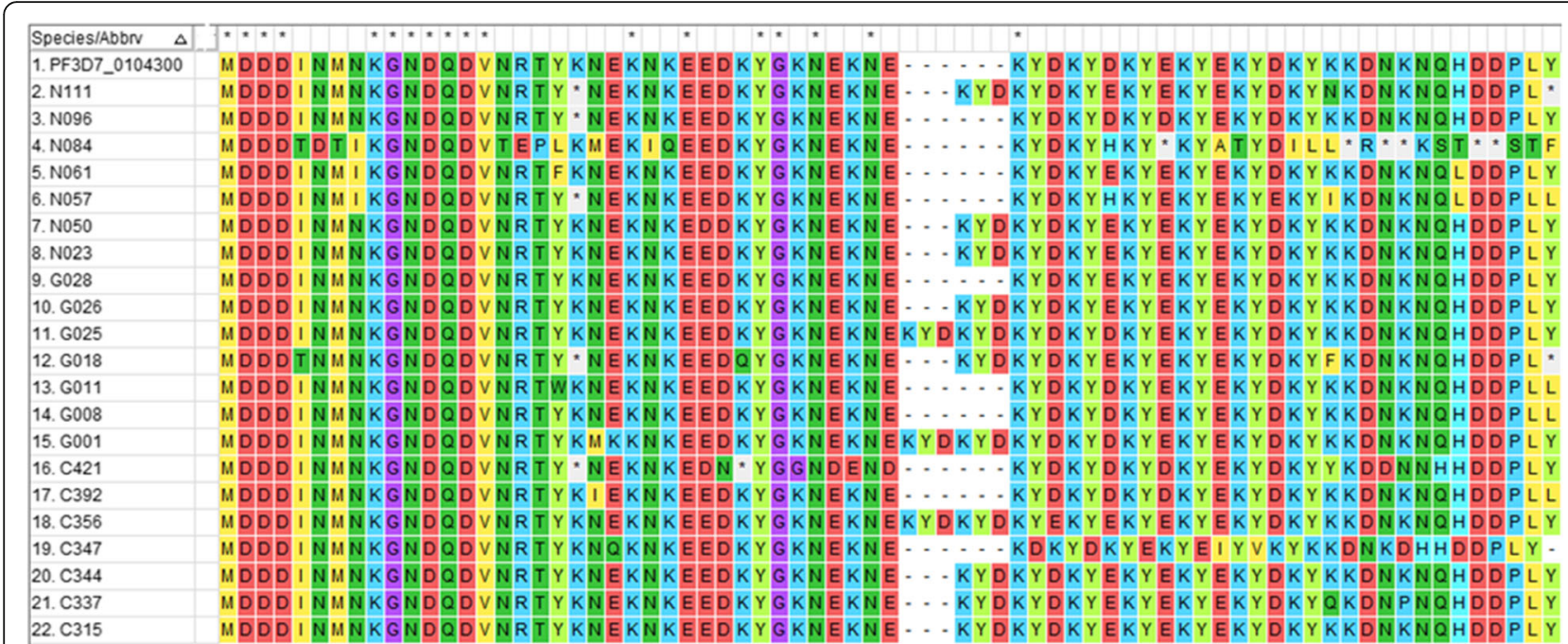

Fig. 7 A sequence alignment of pfubp1 gene showing amino acid changes due to single nucleotide polymorphisms. The alignment was done using pfubp 1 reference sequence of the 3D7 strain (PF3D7_0104300). Mutations from codons 1483-1549 at nucleotide positions 4449-4647 is shown. The gaps are a result of the insertions of amino acids which resulted in a frameshift. The gap between $1519 \mathrm{E}$ and $1520 \mathrm{~K}$ are therefore a result of a 6 amino acid insertion in C356, G001 and G025. The known mutations D1525E and E1528D are shown in the isolates N061 and N096 respectively. A frameshift mutation is observed in N084, after codon 1536 as a result of a deletion

diversity in the mutations in Navrongo isolates with 72 different SNPs. Most of the isolates (63\%) had more than one mutation. The high diversity of SNPs in Navrongo, where there is intense seasonal transmission of malaria, is expected. For pfubp1, Navrongo had the most mutations (96\%), followed by Cape Coast (87\%) and Begoro (86\%). It is quite interesting to observe diversity of mutations from both genes from the guinea savannah zone. However, it must be emphasised that transmission intensity does not affect the evolution of resistance but plays a major role in the spread of resistance genotypes [18]. Therefore, discussing our observations along the line of different transmission intensities from the three distinct ecological zones will be premature.

Insertions/deletions (indels) identified in the Ghanaian isolates were similar to those observed in Burkina Faso, Kenya and the UK patients from Liberia and Uganda $[7,8]$. The most common indels, observed in the isolates from the Ghanaian and the other African countries resulted from an insertion of AAT which resulted in an

Table 4 Genetic insertions in the pfubp1 gene of isolates from the study sites

\begin{tabular}{llll}
\hline Sample ID & Amino acids & Insertion & Nucleotide position \\
\hline C315 & KYE & $\frac{\text { AAA TAT GAA }}{\text { AAA TAT GAA }}$ & 4576 to 4584 \\
C337 & KYE & AAA TAT GAA & 4583 to 4591 \\
C344 & KYE & $\frac{\text { AAA TAT GAA AAA TAT GAA }}{\text { AAA TAT GAA }}$ & 4548 to 4556 \\
C356 & KYEKY & GAA AAA TAT & 4588 to 4605 \\
C360 & KYE & GAC AAA TAT GAC AAA TAT & 4583 to 4591 \\
C321 & EKY & GAA AAA TAT & 4590 to 4598 \\
G001 & DKYDKY & 4563 to 4580 \\
G018 & EKY & 4598 to 4606 \\
G025 & KYDKY TAT GAC AAA TAT GAA & 4585 to 4602 \\
G026 & GAA AAA TAT & 4580 to 4588 \\
G034 & YDKY & TAT GAC AAA TAT GAC AAA & 4579 to 4596 \\
N023 & EKY & GAA AAA TAT & 4582 to 4590 \\
N031 & EKY & GAA AAA TAT & 4582 to 4590 \\
N050 & KNE & AAA AAC GAA & TAT GAA AAA \\
N111 & YEK & & 4549 to 4557 \\
\hline
\end{tabular}




\section{Competing interests}

The authors declare that they have no competing interests.

\section{Publisher's Note}

Springer Nature remains neutral with regard to jurisdictional claims in published maps and institutional affiliations.

\section{Author details}

'Department of Animal Biology and Conservation Science, School of Biological Sciences, College of Basic and Allied Sciences, University of Ghana, Accra, Ghana. ${ }^{2}$ Department of Epidemiology, Noguchi Memorial Institute for Medical Research, College of Health Sciences, University of Ghana, Accra, Ghana. ${ }^{3}$ Centre for Tropical Clinical Pharmacology and Therapeutics, School of Medicine and Dentistry, College of Health Sciences, University of Ghana, Accra, Ghana. ${ }^{4}$ Department of Biochemistry, Cell and Molecular Biology, School of Biological Sciences, College of Basic and Allied Sciences, University of Ghana, Accra, Ghana.

\section{Received: 23 November 2017 Accepted: 1 March 2018}

\section{Published online: 12 March 2018}

\section{References}

1. WHO. Malaria Fact Sheet 2017. http://www.who.int/mediacentre/factsheets/ fs094/en/. Accessed 08 June 2017.

2. WHO. Malaria Fact Sheet: World Malaria Day 2016. www.who.int/malaria/ media/world-malaria-day-2016/en. Accessed 22 Nov 2016.

3. Ariey F, Witkowski B, Amaratunga C, Beghain J, Langlois AC, Khim N, et al. A molecular marker of artemisinin-resistant Plasmodium falciparum malaria. Nature. 2014;505(7481):50-5.

4. MalariaGen: MalariaGen Plasmodium falciparum Community Project. Genomic epidemiology of artemisinin resistant malaria. eLife. 2016;5:e08714

5. Ouattara A, Kone A, Adams M, Fofana B, Maiga AW, Hampton S, et al. Polymorphisms in the K13-propeller gene in artemisinin-susceptible Plasmodium falciparum parasites from Bougoula-Hameau and Bandiagara, Mali. Am J Trop Med Hyg. 2015;92(6):1202-6.

6. Kamau E, Campino S, Amenga-Etego L, Drury E, Ishengoma D, Johnson K, et al. K13-propeller polymorphisms in Plasmodium falciparum parasites from sub-Saharan Africa. J Infect Dis. 2015;211(8):1352-5.

7. Henriques $G$, Hallett RL, Beshir KB, Gadalla NB, Johnson RE, Burrow R, et al. Directional selection at the pfmdr1, pfcrt, pfubp1, and pfap2mu loci of Plasmodium falciparum in Kenyan children treated with ACT. J Infect Dis. 2014;210(12):2001-8.

8. Sutherland CJ, Lansdell P, Sanders M, Muwanguzi J, van Schalkwyk DA, Kaur $\mathrm{H}$, et al. Pfk13-independent treatment failure in four imported cases of Plasmodium falciparum malaria treated with artemether-lumefantrine in the United Kingdom. Antimicrob Agent Chemother. 2017;61(3):e02382-16.

9. Henriques G, van Schalkwyk DA, Burrow R, Warhurst DC, Thompson E, Baker DA, et al. The Mu subunit of Plasmodium falciparum clathrin-associated adaptor protein 2 modulates in vitro parasite response to artemisinin and quinine. Antimicrob Agent Chemother. 2015;59(5):2540-7.

10. Wilkinson KD. The discovery of ubiquitin-dependent proteolysis. Proc Natl Acad Sci USA. 2005;102(43):15280-2.

11. Henriques G, Martinelli A, Rodrigues L, Modrzynska K, Fawcett R, Houston $D R$, et al. Artemisinin resistance in rodent malaria - mutation in the AP2 adaptor mu-chain suggests involvement of endocytosis and membrane protein trafficking. Malar J. 2013;12:118.

12. Abuaku B, Duah N, Quaye L, Quashie N, Malm K, Bart-Plange C, et al. Therapeutic efficacy of artesunate-amodiaquine and artemetherlumefantrine combinations in the treatment of uncomplicated malaria in two ecological zones in Ghana. Malar J. 2016;15(1):6.

13. Duah NO, Matrevi SA, de Souza DK, Binnah DD, Tamakloe MM, Opoku VS, et al. Increased pfmdr1 gene copy number and the decline in pfcrt and pfmdr 1 resistance alleles in Ghanaian Plasmodium falciparum isolates after the change of anti-malarial drug treatment policy. Malar J. 2013;12:377.

14. Price RN, Cassar C, Brockman A, Duraisingh M, van Vugt M, White NJ, et al. The pfmdr1 gene is associated with a multidrug-resistant phenotype in Plasmodium falciparum from the western border of Thailand. Antimicrob Agent Chemother. 1999;43(12):2943-9.

15. Price RN, Uhlemann AC, Brockman A, McGready R, Ashley E, Phaipun L, et al. Mefloquine resistance in Plasmodium falciparum and increased pfmdr 1 gene copy number. Lancet. 2004;364(9432):438-47.
16. Wilson CM, Volkman SK, Thaithong S, Martin RK, Kyle DE, Milhous WK, et al. Amplification of pfmdr 1 associated with mefloquine and halofantrine resistance in Plasmodium falciparum from Thailand. Mol Biochem Parasitol. 1993;57(1):151-60.

17. Golassa L, Kamugisha E, Ishengoma DS, Baraka V, Shayo A, Baliraine FN, et al. Identification of large variation in pfcrt, pfmdr-1 and pfubp-1 markers in Plasmodium falciparum isolates from Ethiopia and Tanzania. Malar J. 2015;14:264.

18. Hastings IM, Watkins WM, White NJ. The evolution of drug-resistant malaria: the role of drug elimination half-life. Phil Trans R Soc Lond B Biol Sci. 2002; 357(1420):505-19.

19. Ng PC, Henikoff S. Predicting deleterious amino acid substitutions. Genom Res. 2001;11(5):863-74

20. Kumar P, Henikoff S, Ng PC. Predicting the effects of coding non-synonymous variants on protein function using the SIFT algorithm. Nature Protoc. 2009;4(7): 1073-81.

21. Kimchi-Sarfaty C, Oh JM, Kim IW, Sauna ZE, Calcagno AM, Ambudkar SV. A "silent" polymorphism in the MDR1 gene changes substrate specificity. Science. 2007;315(5811):525-8.

22. Borrmann S, Straimer J, Mwai L, Abdi A, Rippert A, Okombo J, et al. Genome-wide screen identifies new candidate genes associated with artemisinin susceptibility in Plasmodium falciparum in Kenya. Sci Rep. 2013;3:3318.

23. Kwansa-Bentum B, Ayi I, Suzuki T, Otchere J, Kumagai T, Anyan WK, et al. Plasmodium falciparum isolates from southern Ghana exhibit polymorphisms in the SERCA-type PfATPase6 though sensitive to artesunate in vitro. Malar J. 2011;10:187.

\section{Submit your next manuscript to BioMed Central and we will help you at every step:}

- We accept pre-submission inquiries

- Our selector tool helps you to find the most relevant journal

- We provide round the clock customer support

- Convenient online submission

- Thorough peer review

- Inclusion in PubMed and all major indexing services

- Maximum visibility for your research

Submit your manuscript at www.biomedcentral.com/submit
) Biomed Central 